\title{
Choosing Mars-Time: Analysis of the Mars Exploration Rover Experience
}

\author{
Deborah S. Bass \\ NASA Jet Propulsion Laboratory \\ California Institute of Technology \\ Mail Stop T1718 \\ 4800 Oak Grove Drive \\ Pasadena, CA 91109 \\ 818-393-5189 \\ Deborah.Bass@jpl.nasa.gov \\ Roxana C. Wales, SAIC NASA Ames Research Center \\ Valerie L. Shalin, Wright State University
}

\begin{abstract}
This paper focuses on the Mars Exploration Rover (MER) mission decision to work on Mars Time and the implications of that decision on the tactical surface operations process as personnel planned activities and created a new command load for work on each Martian sol. The paper also looks at tools that supported the complexities of Mars Time work, and makes some comparisons between Earth and Mars time scheduling. ${ }^{1}$
\end{abstract}

\section{TABLE OF CONTENTS}

1. INTRODUCTION: WHAT IS MARS-TIME?....5. 1
2. MARS TIME TRADE STUDY

Considered Staffing Options ............................................. 3

Evaluation Metrics ........................................................ 3

Earth Time - Prime Shift ................................................. 3

Other Components of the Trade Study........................... 4

Trade Study Conclusions and Recommendations ............ 4

3. MISSION EXPERIENCE OF MARS TIME.................4

Local personnel vs. Remote personnel.............................. 4

Orbiting Spacecraft and Data Return Complexity............ 6

4. KEEPING TRACK OF TIME 6

5. EXTENDED MISSION OPERA TIONS

Compressed Planning.

8

Chasing the downlink data return

6. Conclusions

Implications for Mission Management.

7. REFERENCES

8. BIOGRAPHY

\section{INTRODUCTION: WHAT IS MARS-TIME?}

NASA's Mars Exploration Rover (MER) Project successfully landed two spacecraft carrying identical rovers on opposite sides of the surface of Mars in January and February 2004 and conducted two nominal surface missions staffed on "Mars Time" for 90 Martian days each [1]. A

\footnotetext{
${ }^{1}$ 0-7803-8155-6/04/\$17.0002004 IEEE
}

Martian day, or "sol", is thirty-nine minutes longer than an Earth day. Mission planners chose a Mars Time staffing concept to optimize the work of the solar powered rovers and maximize precious rover time in the Martian environment. Each day surface operations personnel produced a planned set of activities and associated commands that were sent to the rovers so that they could do their work of data collection beginning on the following morning at rover wake-up.

Figure 1 (below) shows the Mars Exploration Rover mission timeline that begins with the receipt of data from the spacecraft and completes with a set of commands approved and radiated to the spacecraft for execution the following morning. The time system portrayed in this figure is Mars Local Solar Time (MLST), approximately 40 minutes longer than an earth day. In the chart, action steps are represented by royal blue bars, and meetings and handovers are represented by green bars. The grey bar indicates the time when the rovers execute commands on the Martian surface. In the Mars time scenario, the Earth-based team works while the rovers sleep. Light blue represents the window in which data will be returned from Mars. While the light blue bar appears long, it represents a window in which a 30-minute transfer of data will occur. The time that data is returned varies within the window, and is predictable several days in advance.

Figure 1 allows for the worst-case scenario with regard to late data arrival leaving enough time to ensure that the Earth-based team can produce the necessary results in the shortest time possible. Data can return to Earth either directly from the rovers on the planet surface or from spacecraft currently orbiting Mars. The four vertical bars at the top represent all options for returning relayed data through orbiting spacecraft; the light blue bar represents data returned directly from the planet's surface. Four relay possibilities are described but not all are routinely used each sol. The 10-hour "downlink shift" staff analyze the received data and make recommendations for rover activity execution, while the two 10-hour "uplink shifts" turn the 
recommendations into commands the spacecraft can understand. The process, as planned, originally took 19 hours to complete; by the beginning of extended mission operations, with improvements in automation, increased staff training and expertise development, the process took an average of 8 hours.
This paper focuses on the MER mission decision to work on Mars time, the implications of that decision on the tactical surface Mars time work, the support needed from related tools and the impact that Mars time had on the work of personnel as they planned activities and created a new command load for each Martian sol.

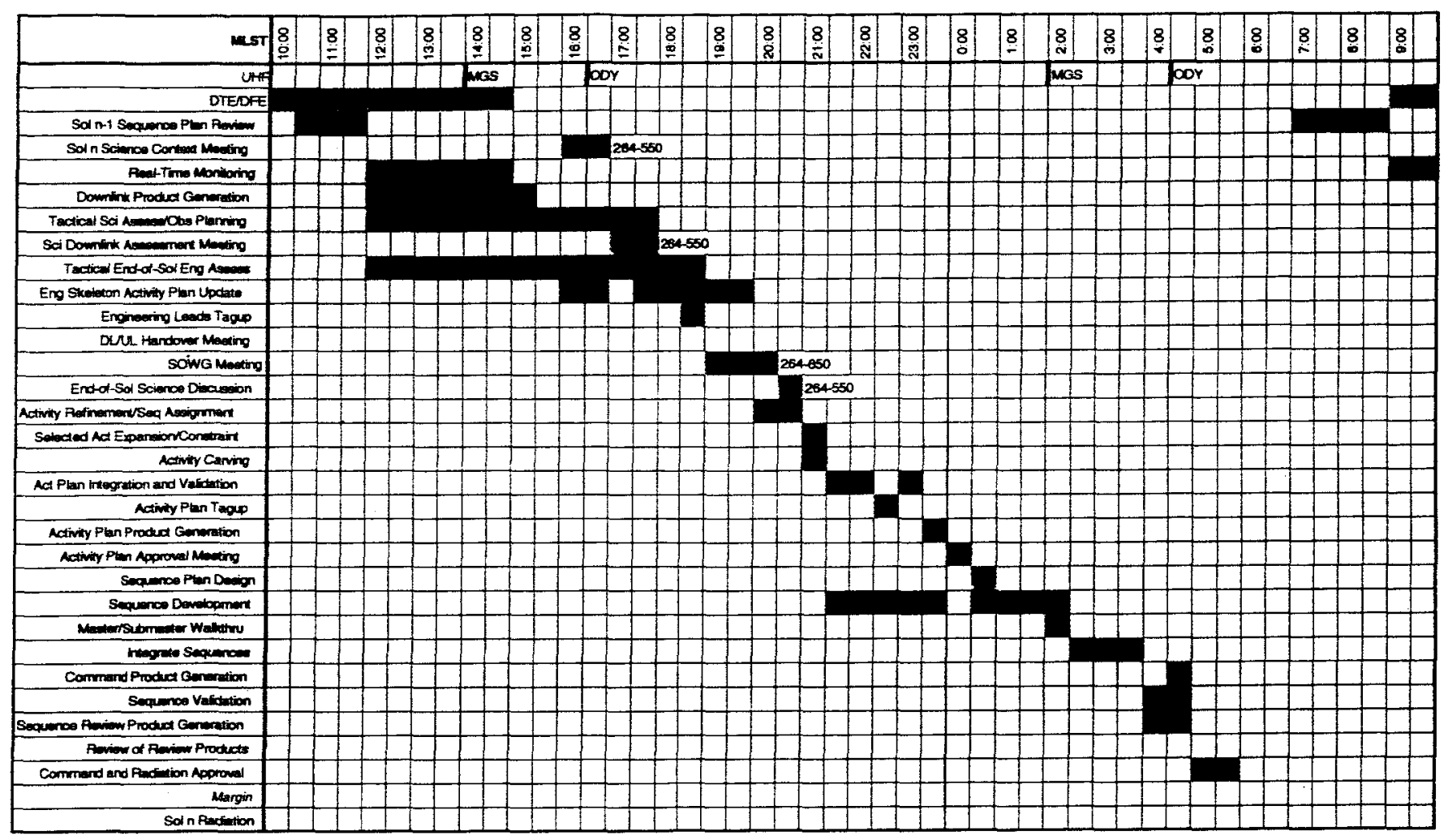

Figure 1. Mission Timeline detailing steps between receiving data from the spacecraft and sending a complete set of instructions to the rover (Courtesy J. Callas)

Because the Martian day is thirty-nine minutes longer than an Earth day, participants who worked on Mars Time reported to work later each Earth day in order to remain synchronized with the solar powered work of the rovers were doing. For example, a participant might report to work at 12 noon on one day, 12:39 the next day, 1:18 the next day and so on. In addition to the daily, or "soly," tactical process of planning and commanding on Mars, other mission work, known as the strategic process, focused on planning over longer periods of time and was responsible for long term mission activities and resource allocations. The strategic processes included coordination of the work of the two missions, coordination and allocation of Deep Space Network and orbital communication coverage within and across missions, determination of overall rover resource situations and public outreach activities. Tactical work on MER was done on Mars Time, while strategic work was done on Earth Time.

\section{Mars Time Trade Study}

The case for Mars Time centers on the following argument: a landed spacecraft operated on solar power is a dying asset. The mission duration is limited by the amount of sunlight the solar panels receive. Further, the spacecraft may fail to respond on any given day, and therefore it is critical to maximize the use of that asset. Arguably, Mars-Time optimizes that usage. However, maintaining Mars-Time in an Earth-Time world poses some problems including some typical of shiftwork [2] and some that were a somewhat more surprising.

Early in the development of the mission, in response to a design review, MER management launched a study to help determine whether to use Mars time staffing. They were looking for an optimal operations schedule for coordinating the work of two hundred and fifty mission personnel on 


\section{Other Components of the Trade Study}

MER mission designers also made an effort to gather data on the effectiveness of operating on Mars Time from members of the 1997 Mars Pathfinder mission. Personnel worked on "Mars Time" for roughly 87 days during that mission and it was the only reference mission for such staffing activity. Although there was some variation in recollection among participants, overall most Pathfinder operations personnel, when questioned informally, agreed that the 1997 Mars Time schedule had advantages and disadvantages and the trade-off decisions were difficult. NASA Ames Human Factors personnel made two studies for the mission; one was a recommendation for work based on a review of the physiological factors that contribute to alertness and performance among shift workers and another was a questionnaire filled out by Mars Pathfinder personnel who had gone through the Mars Time staffing experience in $1997[3,4]$.

\section{Trade Study Conclusions and Recommendations}

Mars Time staffing plans consistently scored better than Earth Time for:

- Ability to command every sol

- Optimal time utilization, conservation of margin

- Response to off-nominal situations

- Maximized potential mission return

- Little or no cross training required

However, Mars Time staffing plans scored lower in sustainability:

- Mars Time staffing requires more crews to sustain extended duration operations

- Straight Mars Time can be sustained for short duration ( $<30$ sols) [5].

With respect to short-term mission concerns, Mars-Time appeared to be the most efficient staffing solution: the operations schedule is fixed to Mars Time, which creates the most margin in the day by maximizing the available time. With respect to short-term mission concerns, Mars-Time appears to be the most efficient staffing solution: the operations schedule is fixed to Mars time, which creates the most margin in the day by maximizing the available time. Margin was recognized as a critical component early in the mission, when the team was still learning some of the quirks of the vehicles and tools and processes were still being finetuned. Therefore, the decision was made to operate on Mars Time at least for the short term.

It was recognized that Mars time would not be sustainable indefinitely due to the need for people to interact with families and duties outside of the mission environment; it is not possible to isolate people indefinitely from their broader responsibilities and personal commitments. "In the beginning, the adrenaline is going and everyone is pretty focused. External stresses can be pushed off. But you can't carry that forever." (Andy Mishkin, personal comm.) Indeed, it was recognized that working on Mars Time is a matter of scale and longevity. Therefore, in the long term, the strain on the team would be evaluated, and the switch to an Earth Time schedule would be considered.

The Ames Human Factors reports recommended that strategies be developed by the MER project to maximize performance and minimize risk to mission success and personnel health. In particular, they recommended that staff members work no more than $\mathbf{4}$ shifts on Mars Time in a row, followed by several days off to allow enough time to recover from Mars Time operational shifts [3].

Other recommendations from the Human Factors studies included fitting all windows in the mission support area with black out curtains and providing available in-house meal service and nap areas. While these recommendations were instituted, others, such as controlled transportation to and from sleep quarters, proved impractical to implement during the short duration (120 days) of nominal surface operations.

\section{Mission EXPERIENCE Of MARS TIME}

The Mars Exploration Rover mission made a conscious decision to operate on Mars Time, and the resulting experience of the teams varied considerably. Of course, with approximately $250 \mathrm{MER}$ operators working on Mars-Time schedules, not all personnel will respond to the schedule in the same manner. For some, Mars Time was easy to maintain, while others found it to be a very challenging way to live. Many of the experiences we report here are consistent with literature regarding the impact of shift work on daily life [e.g., 2, 6]. Studies show that social-cultural issues are at least as problematic as physiological and cognitive considerations. Therefore, mission risk must be described by both the complexity of resulting operational processes and procedures, and the impact to personnel. We discuss these concerns in light of the MER mission experience of Mars Time.

\section{Local personnel vs. Remote personnel}

"Staff with childless, unmarried orphans." (Kevin P. Talley, personal comm.)

In general, the Mars Time experience of personnel who lived all year long in the JPL vicinity (hereafter referred to as local personnel) was different than that of personnel who moved to JPL for the sole purpose of operating the rovers (hereafter referred to as remote personnel). Notably, one of this paper's authors was local, while the other two were remote personnel who moved to JPL for the nominal mission. The experience of the authors is factored into this 
Earth who would be operating dual rovers on Mars. The main question was whether operations processes should be conducted on an Earth-Time schedule or a Mars-Time schedule. The MER staffing options had to consider the following criteria:

- Allow for personnel to be fully trained for surface operations before landing day,

- Acknowledge that personnel would take longer to do their work early in the mission while they developed expertise,

- Provide the capability to command every Martin day,

- Provide margin for dealing with contingencies within the nominal timeline,

- Minimize the impact of scheduling on personal lives,

- Be sustainable over the life of the nominal mission.

- Ensure communication of key information across shift boundaries,

- Possess resiliency in the face of anomalies,

- Support operations of two rover missions simultaneously,

- Maximize the use of shared facilities, such as meeting rooms and the engineering surface support area,

- Maximize the potential for science return,

- Minimize the risk to the spacecraft.

\section{Considered Staffing Options}

The mission considered a variety of staffing options. We present here an analysis of the two extreme cases.

\section{Evaluation Metrics}

Five functional areas for metrics were identified, and a relative weighting for each area was applied

- Number of Personnel

- Impact on Facilities

- Impact on Personnel Morale

100

- Complexity of Process

150

- Achieve full Mission Return

400

- Impact of other Human Factors

100

A perfect score for a particular staffing option would total 1000 points.

We examine the possible staffing options using the following set of common terms. "Commanding every sol" describes the ability to affect the plans for sol-n+l using the results from sol-n. The term "core sleep" refers to a set of Earth hours in the middle of the night preserved for human sleep every day. This start time of the core sleep hours does not have to be the same for all shifts, but each shift must have a minimum number of hours off in the middle of the Earth night. "Shift, fixed" suggests the time period and length a person or crew is expected to work, fixed to Earth time. "Shift, floating" refers to the time period and length a person or crew is expected to work, floating with respect to Earth Time. "Shift, rotating" defines the minimum number of people who can evaluate a downlink, receive additional input from the strategic process, and generate an uplink with a 15-hour period. "Members of a squad", by definition, require training in only one key task.

Baseline Mars Time-The baseline Mars Time option focuses staffing resources both in Time and with regard to tasking. On an assigned workday, each person comes to work at approximately the same Mars local time and does whatever s/he does best. Key characteristics of this option include: at least one opportunity to uplink commands to the spacecraft each day, 4 hours of margin in the schedule, personnel work the same functional position each duty cycle, shift start moves 39 minutes later each day while the operations tasks stay synchronized with the Mars clock, as do individual work schedules. Shift overlap from downlink to first uplink shift (Uplink1) was approximately one hour, overlaps from Uplink1 to Uplink2 was 1 hour, whereas Uplink2 to Downlink shift did not overlap. (Note that this absence of overlap was ultimately deemed unacceptable to MER management, and key operations staff were placed so that there was always face-to-face handovers of information between all shifts.) Shift handovers always occurred at the same point in the process. This option did not necessitate "split shifts" where an operator finished the second part of his or her shift the following morning. Calculations indicated that this schedule would include one third night shifts over the duration of the 90 day mission, Because the operations area would be continuously staffed in this option, the ability to respond to off-nominal situations and respond to ad-hoc science team requests was maximized.

\section{Earth Time - Prime Shift}

This option employs a single long shift each day (up to 8 hours), so that the rover lacks a ground team "babysitter" for as many as 16 hours per day. People keep the same time schedules each day and stop at the end of the shift, independent of Mars Time. Only eight hours between the Mars afternoon downlink and the uplink the following Mars morning can be populated with rover activities, in general, and sometimes as little as 3 hours. Shifts are fixed times, and work flows over to next day, whatever its status. Cross training is essential for this option, as personnel duties must support the schedule of the rover on Mars Time, and staff must be capable of some productive activity during any phase of mission operations. The ability to command every sol is precluded because a shift may not be able to prepare an uplink plan within the available Earth-based work window. This option is based on the concept that the process will allow commanding as often as possible but that commanding will be limited to the ability to complete work within the Earth time schedule. 
analysis as the authors were both observers and experimental subjects in the topic of Mars Time operations.

Personnel who moved temporarily to Pasadena from remotely located institutions, most specifically the science team, generally had less difficulty working the Mars Time schedule. Special housing arrangements at local apartment complexes provided furnished apartments with black-out shades and flexibly scheduled cleaning services. However, as one team member commented, "It was difficult for housekeeping to understand that I really didn't want to be disturbed from 8 am to $4 \mathrm{pm}$ every day." (Pancam team member, personal comm.)

The experience of JPL local personnel differed from person to person, but overall, they found that Mars Time significantly impacted their personal lives. Because local personnel also had to manage time with their families, the added personal stress and conflict lead to a greater level of fatigue. For personnel who were married and both partners working MER, the situation was extremely stressful, as often there was no one left at home to manage daily tasks such as child and/or pet or parent care. At least one MER operator rented an apartment close to JPL and lived apart from his family when he worked MER shifts. He spent his days off in his home with his family, much like individuals who commute and live between two cities.

The fatigue of Mars-Time may create risk and slow mission work and activity in daily life. One of the primary consequences of mental fatigue is that judgment is impaired, and affected individuals are not capable of making sound choices for their own well-being [6]. One local team member was injured after a series of Mars time shifts when he mistakenly walked into a wall. Another local team member reported falling asleep at the onramp to the freeway. Fortunately, the authors know of no significant or long-lasting injuries resulting from the MER Mars Time experience. Arguably, these kinds of issues are regularly seen in professions that employ overnight and long shift work, and cannot be attributed to the Mars Time staffing plan alone.

In contrast to remote personnel who temporarily relocated, local JPL personnel were sometimes allocated to a second task along with working operations for MER. Local personnel had to continue to attend institutional meetings. Personnel often ended up staying long after a shift to attend Earth time meetings related to other aspects of their work life. Operations staff also came in on their "days off" in order to attend to these kinds of tasks. Finally, professional travel, to conferences for instance, during MER Mars time operations was difficult to schedule at best. Travel planning was challenging for all team members, as they were living on Mars time but had to insert themselves in established Earth time flight schedules.
Local personnel also had to continue to fill out time cards. However, JPL time-keeping systems were not flexible enough to schedule more than 40 hours of work time, with 8-hour days. The time-keeping system could also not respond to the problem of operators working 4 days on, 3 days off, without a 2 day break for weekends. Negotiations were held, and this problem was eventually surmounted on a case-by-case basis.

\section{Concessions and Adaptations}

Working on Mars-Time carried with it a notion that it was a "badge of honor". People who were not ready to sign up for Mars-Time were not truly committed to the project. This social status made working Mars-Time hard to resist. "I'm working Mars time all the time" was interpreted as staff members who had committed completely to the project. However, concessions were required, creating a number of special cases.

Some operations teams scheduled "discretionary" work time where staff was expected to be at work but not necessarily operating a rover. In some cases, particular staff members' needs were accommodated, such as the preference of one member to start and end his shift at as close to the same times of day as possible. This staff member rotated between spacecraft when the start and end times moved too far out of an acceptable range. Another staff member was uncomfortable working nights, so accommodations were made so that he worked as many day shifts as possible.

A schedule that mandated multiple, consecutive rest days was implemented to facilitate recovery from Mars Time operational shifts [e.g., 3]. However, the various operations teams implemented the schedule differently. One team chose to work 4 days on shift, 3 days off shift. Another team worked 7 days on, 7 days off. Some personnel stayed on Mars time on their days off (Brian Chafin, personal comm.) while others moved back to Earth Time on their days off. Some personnel attempted live both Mars and Earth lives with limited success.

It is because of the variation in implementation of Mars time that it is difficult to provide a single coherent story regarding the effects of this staffing option.

Gastric distress is a main symptom of shift work, but it may very well be a side effect of this work, because it is difficult to know what meal to eat and when to eat it when starting a 2:30 AM shift. Eating times were personal and there were limited opportunities to share meals. Fresh, healthy food was not as readily available in the middle of the night. JPL meal services had to be modified significantly to accommodate Mars Time work, as there were few food resources in the nearby area around the clock. Many operations staff brought large amounts of food with them and stored snacks and frozen foods in project-provided kitchen facilities. Free ice 
cream was also available to the team at all. Because the ice cream was easily available, operations personnel ate more of it than they would have normally (3-5 ice cream bars/day was not unusual). Some people gained weight. There is anecdotal evidence that team members relied on the ice cream as both a reward and a pick me up to push through the harder parts of their shift work.

The majority of issues described thus far reflect issues currently in the shift work literature. We turn now to a set of issues unique to Mars time and the shifting asynchrony between Earth time and Mars time.

\section{Orbiting Spacecraft and Data Return Complexity}

In some respects, Mars time appears to have simplified the complexity of the operational processes. Mars time reduced risk by maximizing the time available to generate a complete command cycle. Mars time allowed for more frequent direct communication interactions between the ground operations team and the spacecraft. When rovers exhibited anomalous behavior, Mars time simplified the team's ability to respond. Key personnel could extend their shifts to resolve problems. Finally, both the clock and direct communication passes (DTE) were in synch with the work.

Mars time also made it possible for personnel to understand what was currently (taking into account the one-way light time travel delay for returned information from Mars) occurring on the spacecraft. It remains to be seen whether this state knowledge is always necessary to the successful operation of a spacecraft, but working in synch did help the operations team establish a bond with the vehicles on Mars.

The original MER mission timeline was designed to utilize direct to Earth (DTE) communications, which was expected to provide the data necessary to make each sol's decisions. However, most data for MER was relayed to the Earth team through orbiting Martian satellites rather than DTE, The satellites had to be in accurate orbital positions to capture the data from the Martian surface as well as to transmit it to Earth. Transmission latency occurred as the satellites orbited to re-position themselves for each of these tasks.

On Mars Time, the assumption was that the DTE data would be available to make decisions for each sol. While the team was not supposed to hold decision processes for the return of particular data, the natural inclination was to hold off on decision-making where possible so that the most recent information could be incorporated. Because most data were returned by relay, the team often waited for this data to be returned before making decisions. This had the effect of sometimes delaying the process, resulting in last minute decision making and pressuring the team to complete tasks in shorter than planned times.

During operations, data return times were not set on precise schedules but rather occurred during "windows" of opportunity, Mars time staffing could not decrease the complexity introduced by these transmission latencies. For example, on each sol data was examined when the operations staff came to work on their Mars Time shift. This might be hours after the actual receipt of the data by the mission. If the time differential between receipt of data and data analysis was particularly extensive, it could draw down margins in the daily planning and command load cycle causing pressure on the timeline. Latency made it much more difficult to optimize data return events.

In section 5, we discuss the relationship between Earth Time staffing (during MER extended mission operations) and data return latency.

Mars Time personnel worked schedules that were difficult to remember and predict beyond a day or two in advance without the aid of carefully prepared electronic schedules. We will address in detail the issue of keeping track of schedules as well as several different times in Section 4.

\section{KeEPING TraCK OF TIME}

MER mission personnel working on Mars Time had to keep track of several different kinds of time and coordinate those times with work staffing schedules. Some of the different time and coordination issues were:

- Tracking Mars time within a mission in order to plan rover activities relative to Martian daylight and send commands to the rovers in a timely fashion.

- Tracking both Mars Time and the equivalent Earth Time in each mission so that personnel could keep track of events inside and outside the mission while they were working.

- Coordination of time between the two missions for sharing Mars communication infrastructures and sharing mission facilities.

- Scheduling of work within a sol so that participants completed each phase of work and handed over to the next team within the mission timeline of events.

- Scheduling of work to identify both the consistent Mars start time for shifts as well as the constantly rotating Earth start times for the same shift so that personnel knew when to show up for work.

- Identification of long term staff schedules so that personnel could keep track of their schedules relative to both Earth and Mars Time over extended time periods and when planning for days off.

Working on Mars time, personnel had to track both the Martian local solar time (LST) as well as the Martian date (Sol number) and coordinate those times with Earth Pacific Standard Time (PST), the Universal Time (UTC) also 
known as Greenwich Mean Time (GMT) and the current Julian calendar Earth day and date.

The two rovers were working on opposite sides of Mars, at the Meridiani Planum and Gusev Crater sites. While this difference facilitated easier recollection of rover-specific issues, it doubled the time challenges as the work of the two rovers took place in different Martian time zones, MER A, the Spirit mission at Gusev Crater, and MER B, the Opportunity mission at Meridiani Planum, had different times known as MERA LST and MERB LST. Whether personnel were working on the Spirit or Opportunity missions, they had to know what time it was on their particular area of Mars and make translations between Mars and Earth time with ease. While daylight and darkness helped staff keep track of Earth time to some degree, there were no environmental clues to help track Mars time.

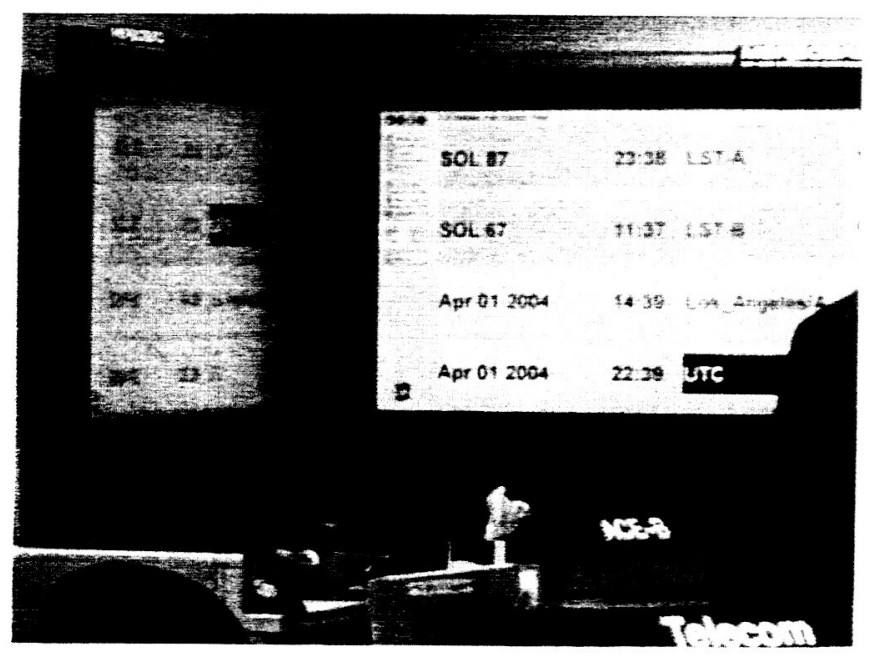

Figure 2. CIP clock tracks LSTA, LSTB, PST and UTC times.

Additionally, science team personnel who had moved to Pasadena for the duration of the mission from an institution in another time zone had to keep track of the time in relation to their "home" time zone. A mission tool, the Collaborative Information Portal [7] had clocks that were designed specifically to allow personnel to configure and track all of these times as needed. For instance, participants could track and compare LST A and/or LST B, track and compare Mars Time with PST or with time in another Earth time zone, and track UTC (universal time coordinated). Images one and two below show two such configurations of the CIP clocks displayed on another mission tool the MERBoard [8]. The large display of these configurable clocks on the MERBoard became an essential work practice, facilitating work on the mission time line (Figure 2 and 3 ).

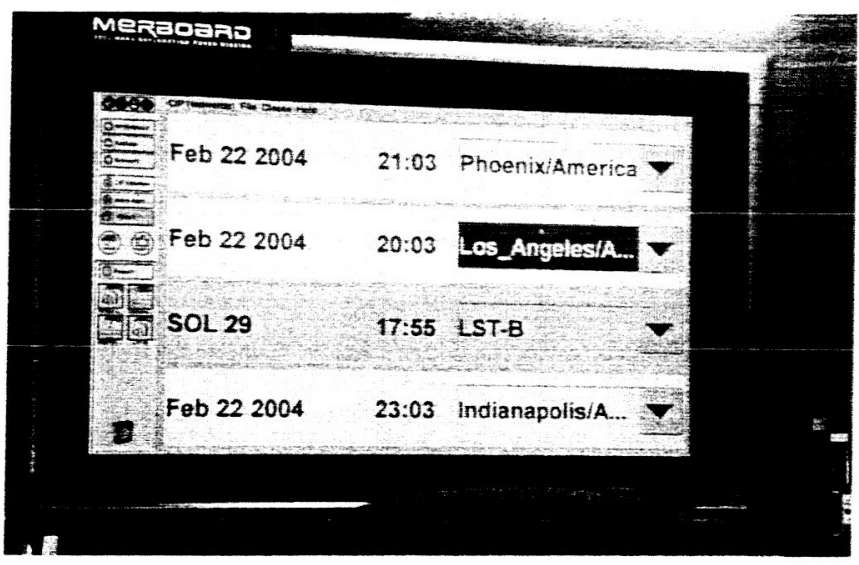

Figure 3. Software clock tracks LST and PST with other Earth time zones.

Staffing schedules had to indicate both Earth and Mars shift start times. While the start of Mars time work might always be $1400 \mathrm{hrs} \mathrm{LST}$, the fact that the related Earth time start of work "walked forward" by thirty nine minutes each day, forced teams to create elaborate schedules to coordinate work. While it was intuitive to personnel that they needed to report approximately forty minutes later for work tomorrow, it was not intuitive or easy to track a thirty nine minute change over even short periods or when planning events several days or weeks ahead. It was also extremely easy to lose track of the time rotation when taking days off between work periods. Even just a few days off made it difficult to project ahead and determine Earth time relevant to a particular time on the Martian clock. For example, to ensure getting to work on time, two of the authors felt compelled to write start times for every Mars day on their Earth calendars and consider the changing traffic patterns in deciding on when to leave for work. Further, updates to schedules were difficult to track. Often, email was used to distribute versions of the staffing schedules, which suggests that staff members must check their email at the right time (either at home or at work) to ensure that they were aware of schedule changes.

Many operations team members worked more than one position and thus had schedules from more than one position manager. It was difficult for personnel to coordinate these schedules across positions. Although team members recognized the need to create an integrated scheduling and staffing tool, higher priority concurrent hindered the implementation. 


\begin{tabular}{|r|c|c|c|c|}
\hline \multicolumn{1}{|l|}{ MER A } & \multicolumn{5}{|c|}{} \\
\hline $\begin{array}{c}\text { Date [PDT] @ } \\
\text { PDL Shift Start }\end{array}$ & $1 / 5 / 04$ & $1 / 6 / 04$ & $1 / 7 / 04$ & $1 / 8 / 04$ \\
$15: 08$ & $15: 48$ & $16: 27$ & $17: 07$ \\
\hline Approx. Sol & 3 & 4 & 5 & 6 \\
\hline Pancam PEL & JBe & JBe & JJo & JJo \\
\hline Pancam PDL & $\mathrm{MLe}$ & $\mathrm{MLe}$ & $\mathrm{RMo}$ & $\mathrm{WFa}$ \\
\hline Pancam PDA & $\mathrm{JSO}$ & $\mathrm{FSe}$ & $\mathrm{FSe}$ & $\mathrm{MJO}$ \\
\hline Pancam PDA2 & $\mathrm{MWO}$ & $\mathrm{WFa}$ & $\mathrm{MJO}$ & $\mathrm{JSD}$ \\
\hline Pancam PUL & $\mathrm{JPr}$ & $\mathrm{JPr}$ & $\mathrm{HAr}$ & $\mathrm{HAr}$ \\
\hline Pancam PUL2 & $\mathrm{EMc}$ & $\mathrm{MBM}$ & $\mathrm{JPr}$ & $\mathrm{MBM}$ \\
\hline
\end{tabular}

Figure 4. Section from one of the science team schedules showing mission (MER A), staff position, Earth Time and date as well as Martian Sol when shift starts. (Courtesy of Jim Bell and the MER Pancam team)

Many mission participants invested in special analog watches, called "Mars watches," that were adjusted to keep Mars Time. The watches were extremely helpful, because a glance told personnel that they had three Mars hours before they had to show up at a 1400 LST meeting or start of shift. The limitation of the watches was that if one went off Mars Time for several days and did not pay attention to mission events, it was impossible to know if the analog watch, without a 24 hour time display, was indicating morning or evening times, One could not just look out the window and tell if it was dark or light on Mars. In general, use of Mars time reduced the cognitive load on mission personnel because the team did not have to translate task completion times into rover need times. But participants still had to translate time differences because alarm clocks run on Earth time and the analog Mars watches did not have alarms.

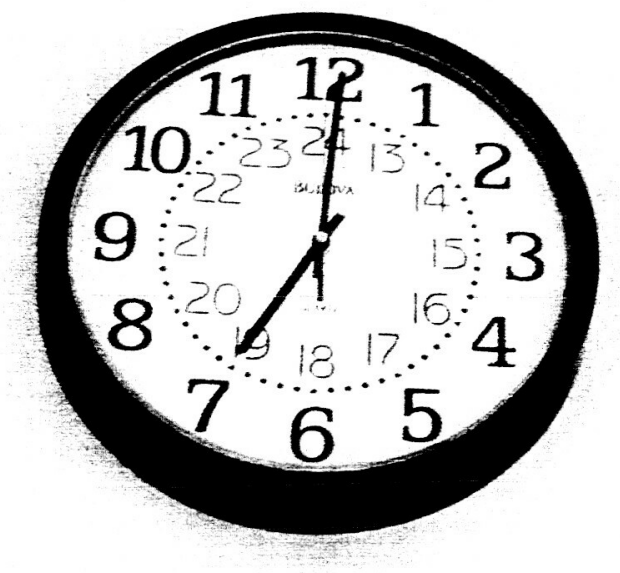

Figure 5. Since mission Earth Time was kept in military time, workers also had to translate PST to military time. A few clocks in the mission support facility helped with this coordination.
While some analog wall clocks displayed both military and PST (Fig 5), they did not indicate whether the Earth time of day was morning or evening, 0700 or $1900 \mathrm{hrs}$, or whether it was day or night on Mars. The number of necessary, relevant time conversions at each point of work in the Earth day and Martian sol was complex.

\section{EXTENDED MISSION OPERATIONS}

The MER mission moved from nominal to extended mission operations after successfully working for ninety days/sols (the definition of mission success) with each rover. At this time, the teams also transitioned to "Earth Time schedules". With the onset of extended mission, many science team members also returned to their home institutions creating distributed work and teams. Based on the switch to Earth Time and the nature of extended operations, we can look at the operational effects of Mars Time from yet another perspective.

During the nominal mission, science and engineering participants could refer to a paper schedule that, once determined, rarely, if ever changed unpredictably. With the shift to Earth Time operations, MER teams still had to work in relation to the timing of pre-existing Mars events, including uplink and downlink opportunities. This precluded simply moving to permanent, regularly scheduled Earth business hours. Instead it required the mission to create shifting schedules that allowed people to work in Earth time but still maximize synchrony between Earth and Mars Time. Because the shifting schedules were unpredictable, local and remote participants had to check an e-mail schedule to confirm meeting times. However, participants could not be sure that they had received the most recent update in their email, creating substantial opportunity for confusion. Shifts started later over time, but it was not clear on what day the shift would move to a later time.

Science teams consolidated the work of both rover operations within a single daytime schedule, offering them the opportunity to unite in a joint meeting. However, on occasion, managing the asynchrony for both rovers made it impossible to identify a feasible joint meeting time, again contributing to schedule uncertainty.

In order to coordinate the drifting Earth and Mars times, at some point in the shifting schedule process, a dramatic resynch was required. Instead of starting the day later, participants would start the day much earlier. These days were called "transition days", and potentially reversed the order of activities. Prior to transition, downlink may have occurred at the end of the shift. When modest shifting could no longer keep the downlink in the window of one workday, transition days restored the downlink event to the beginning of the shift. If the timing of the communication event varies within the workday, the organization of the related work will 
also vary. A new type of (sol) also emerged. So-called "restricted sols" are those in which downlink is not available before planning so planning had to occur two sols ahead.

\section{Compressed Planning}

The shift from Mars Time to earth Time also required a compressed science planning process. This affected the planning process in many ways.

\section{Chasing the downlink data return}

When staffing to Earth Time, the issues related to data latency take on a different character. On Earth Time, there was no expectation that the downlinked data would arrive in time to influence the decision process. Therefore science planning was less dependent upon immediately-returned data and instead relied on the data already in hand. We argue that Earth Time staffing makes the planning more realistic by not expecting the team to perform " $11^{\text {th }}$ hour miracles" that require the completion of tasks in far less time than called for in the process.

When the vehicles first began operating on Earth Time, the Opportunity science team appeared to be chasing the downlinks, postponing the refinement of a single plan until after a crucial planning meeting (the SOWG) in an attempt to continue the practice begun in the nominal mission of waiting for downlink information. As the time of downlinks continued to diverge from the work of Earth time operations, however, this strategy was not sustainable. Adaptations in response to late arriving data could not be incorporated into the planning process with sufficient speed to meet the uplink so participants began to move from reactive planning to predefined, relatively context independent, multi-sol plans. Planning began before the arrival of new data, resulting in more generic plans. However, during periods of heightened interest, the science team reverted to semi-Mars Time in order to react to the latest data.

\section{Conclusions}

The MER mission's desire for complete flexibility in sequencing the rovers on Mars, as well as the ability to maximize the availability of co-located operations personnel led to the decision to staff on Mars Time. The nature of future missions however, will dictate different assumptions about the nature of tele-operations, including the length of the mission, the particular software tools being used, and the nature and amount of autonomy being used. It is likely that each mission will consider these assumptions relative to any possible staffing options when deciding on the nature of tele-operations of remote spacecraft. Depending on each missions design, it may not be necessary to work on Mars Time, despite the obvious mission advantages to MER.
We suggest that if MER had had the resources (primarily time) to significantly cross-train the operations team on both uplink and downlink tasks, then much of the need for Mars Time staffing could be eliminated. Without cross training, the schedule determines the nature of work available to a particular operations staff. Operations might still require 24 hours/day staffing, but personnel could maintain a regular shift schedule and perform whichever task was required on Mars when they came to work on their Earth Time schedule. In future long-duration, extended ( 1 year and more) missions, the staff cross-training should be possible.

Additionally, we suggest that deficiencies in automation, tool integration and process optimization show their impact in staffing. That is, if the process and tools do not support a short turn-around, then it falls to Mars Time staffing to maximize the time available to move from data-receipt to command-upload. If the process for a complete turn around were shorter, the staffing profile would not be the issue; the team could accomplish what is necessary on an Earth Time schedule.

\section{Implications for Mission Management}

A number of institutional challenges should be addressed when considering Mars Time staffing.

- Institutional time keeping policies and food services must be negotiated to accommodate the 24-hour, 7 days per week staffing.

- While schedule personalization is a significant challenge faced by management in charge of scheduling, it is unlikely that all customization can be eliminated. Mechanisms such as scheduling tools should be considered to help address this issue.

- Better mechanisms for schedule distribution should be considered. Email distribution is limited by issues of server latency, versioning, and intermittent participant access to emailed notifications.

- Management should also consider scheduling "discretionary" time as part of the mission work time so that personnel have Earth daytime hours to complete other non-operations work such as attending staff meetings and seminars. 


\section{REFERENCES}

[1] Steven W. Squyres, Ray E. Arvidson, J. F. Bell III, Johannes Bruckner, Natalie A. Cabrol, Wendy Calvin, Michael H. Carr, and the Athena Science Team, "The Spirit Rover's Athena Science Investigation at Gusev Crater, Mars", Science, August 6, 2004.

[2] Donald I. Tepas, Michael J. Paley and Stephen M. Popkin, "Work schedules and sustained performance." in G. Salvendy (ed.) Handbook of Human Factors and Ergonomics (pp. 1021-1058), New York: J.Wiley, 1997.

[3] Charles A. Czeisler, Mary A. Carskadon, Claude Gronfier, Thomas Roth, Melissa M. Mallis, and Kenneth Wright, "Consultant Report: Mars Exploration Rover Surface Operations Human Factors Workshop", NASA Ames Research Center commissioned for NASA Jet Propulsion Laboratory, California Institute of Technology, January 10,2001

[4] Bonny Parke, Michael Shafto, Jay Trimble and Roxana Wales, Mars Pathfinder Fatigue/Stress Questionnaire Report, NASA Ames Research Center, August, 2001.

[5] Arthur Thompson, Deborah Bass, Charles Budney, Karen Deutch, Robert Gounley, Kenneth Herkenhoff, Paul Herrera, Jan Ludwinski, Justin Maki, and Kevin Talley, "Mars vs. Earth Time Final Report", JPL MER internal document, September, 2001.

[6] Bonny Park, "Best Practices in Shift Handover: Applications to MER Surface Operations." Ames Research Center, NASA, MS 262-4, Moffett Field, CA 94035-1000.

[7] Joan D. Walton, Ronald J. Mak, and Leslie F. Keely, "Collaborative Information Portal: MER and Beyond", 2003 Space Mission Challenges for Information Technology Conference, 2003.

[8] Jay Trimble, Roxana Wales, Rich Gossweiler, "NASA's MERBoard: An Interactive Collaborative Workspace Platform". In O'Hara, Perry, Churchill and Russell (eds), Public and Situated Displays: Social and Interactional Aspects of Shared Display Technologies. Dordrecht: Kluwer, 2003.

\section{BIOGRAPHY}

Deborah S. Bass, Ph.D. currently works at the NASA Jet

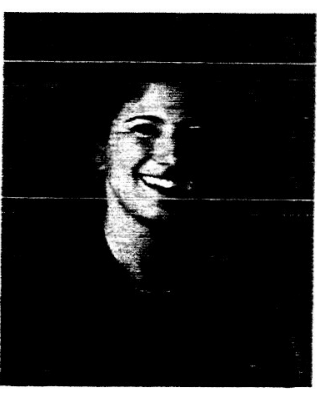

Propulsion Laboratory, where she was the Deputy Science Team Chief for the Mars Exploration Rover (MER) Project. Dr. Bass is now the Project Science Systems Engineer for the 2007 Mars Lander, Phoenix. She received her $\mathrm{PhD}$ from the University of California at Los Angeles, and her science expertise focuses on Mars polar geology, with an emphasis on water transport in and out of the polar regions.

Roxana C. Wales, Ph.D. is a human-centered computing

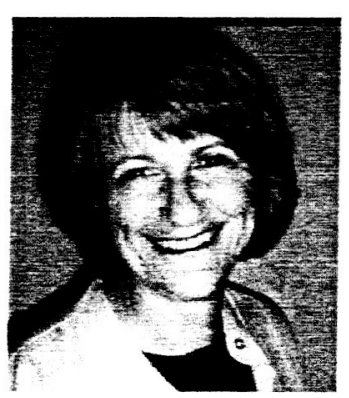
research scientist at the NASA Ames Research Center. As an ethnographer focused on work system design and evaluation, she is especially interested in revealing explicit as well as implicit information in technological settings that is essential for creating better work systems and supporting technology use within expert domains. At NASA, she has worked on projects in Mission Control at Johnson Space Center for both the International Space Station and the Space Shuttle. She also conducted a two and a half year study of airline operations and airline delays at a major airline for NASA.

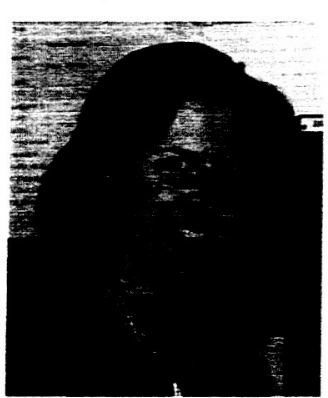

Valerie L. Shalin Ph.D. is currently an Associate Professor in the Department of Psychology at Wright State University, and has held positions at SUNY Buffalo Department of Industrial Engineering and Honeywell Systems and Research Center. She has seventeen journal articles and book chapters, and numerous proceedings papers in the area of human workplace expertise and associated empirical and analytic methods to support the design and evaluation of workplace aiding and training technology.

The research described in this (publication or paper) was carried out at the Jet Propulsion Laboratory, California Institute of Technology, under a contract with the National Aeronautics and Space Administration. 